\title{
Calcium signaling: an underlying link between cardiac disease and carcinogenesis
}

\author{
Xuehong $\mathrm{Xu}^{1 *}$, Steven P. Balk ${ }^{2}$, William B. Isaacs ${ }^{3}$ and Jianjie Ma ${ }^{4}$
}

Cardiac disease and cancer are the top two health threatening disorders, resulting in the loss of millions of lives every year. The countless spending has attempted to mitigate these losses; however, the benefits of this spending do not yet meet society satisfaction. Many research efforts are focused on elucidating the molecular mechanisms underlying carcinogenesis, including PD-1/ CTLA-4, CCR, cytokines, P53, Notch and Wnt signaling pathways. Clinical trials with PD-1/CTLA-4 immune checkpoint inhibitor-based cancer therapy have been especially promising, but a major challenge is autoimmune reactions including mortality due to myocarditis [1]. Thus, more intensive study is needed to examine the impact of cancer treatment on cardiac disease.

Calcium signaling, a fundamental pathway involved in control of cardiac function and cell proliferation and malignancy, might provide a link between cardiac dysfunction and cancer progression and therapy. Calcium signaling is involved at the molecular and cellular levels in most biological processes, such as neuronal excitability, excitation-contraction coupling in the heart and skeletal muscle [2,3], embryo fertilization and development [4], and bone formation and remodeling. Altered calcium metabolism has been linked to tumorigenesis and heart failure $[5,6]$.

Intracellular calcium levels are regulated by the cell membrane calcium-sensing receptor (CaSR), which detects levels of extracellular serum calcium, and inositol 1,4,5-triphosphate receptors (IP3Rs) that regulate calcium release from intracellular stores. This calcium regulation is critical for multiple functions including cell proliferation, apoptosis, fertilization, and development.

\footnotetext{
*Correspondence: xhx0708@snnu.edu.cn

${ }^{1}$ Laboratory of Cell Biology, Genetics and Developmental Biology,

Shaanxi Normal University College of Life Sciences, Xi'an 710062, China

Full list of author information is available at the end of the article
}

In a recent study, Bononi et al. found that the tumor suppressor BAP1, previously characterized as a nuclear deubiquitinase, is also localized in the endoplasmic reticulum (ER). This ER localized BAP1 deubiquitylates and thereby stabilizes ER IP3R3, so that BAP1 loss results in decreased calcium flux and impaired apoptotic responses [5]. In another study Kuchay et al. reported that the F-box protein FBXL2 binds to and ubiquitylates IP3R3, targeting it for proteasome-mediated degradation and thereby limiting calcium influx into mitochondria and apoptosis [6]. They further found that PTEN competes with FBXL2 for IP3R3 binding, so that PTEN loss (in addition to its activation of the PI3 kinase pathway) increases the FBXL2-dependent degradation of IP3R3. In this thematic series, Wang et al. [7] and Xu et al. [8] summarize these recent findings linking the essential role of calcium signaling in normal cells to cancer progression and treatment.

Resident cardiac macrophages have multiple functions including post myocardial infarction repair, which may involve intercellular calcium signaling cross-talk between cardiomyocytes and macrophages. Hulsmans et al. discovered that resident macrophages can communicate with the atrial-ventricular node of the heart to facilitate cardiac electrical conduction [9]. Distinct gene expression patterns related to intracellular calcium signaling could contribute to the inflammatory response and contractile dysfunction of the heart. Moreover, coordinated cell programming may underlie the specific lineage of the resident macrophage in the heart. In this thematic series, Zhou et al. [10] provide a review of the literature that links calcium signaling to diverse functions in different tissues.

Resolving the spatial and temporal aspects of calcium signaling in vivo and in vitro is critical for understanding the physiology and pathology of heart disease and cancer treatment. In this thematic series, Staehlke et al. 
[11] designed a highly sensitive approach with an aminogroup containing plasma polymer nanolayer to examine calcium ion mobilization in osteoblasts. Conjugating near-infrared indocyanine green (ICG) dyes with human serum albumin by covalently binding to gold nanorod, Zhang et al. [12] have developed a sensitive near-field fluorescence detection approach that will provide a more sensitive tool for detecting calcium signaling in vivo at the cellular level and in living animals theoretically.

\section{Authors' contributions}

XHX conceived of the study. XHX, SB, JM and WI prepared the manuscript and edited the manuscript. All authors read and approved the final manuscript.

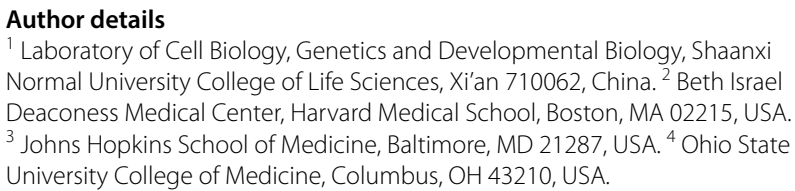

\section{Acknowledgements}

This work was supported by the NSFC (\#31571273/31771277/31371256 to XHX), the Foreign Distinguished Scientist Program (\#MS2014SXSF038), the National Department of Education Central Universities Research Fund (\#GK20130100/201701005/GERP-17-45), and US Maryland Stem Cell Research Fund (2009MSCRFE008300).

\section{Competing interests}

The authors declare that they have no conflict of interest.

\section{Availability of data and materials}

Not applicable.

\section{Consent for publication}

Not applicable.

\section{Ethics approval and consent to participate}

All applicable international, national, and/or institutional guidelines for the care and use of animals were followed.

\section{Publisher's Note}

Springer Nature remains neutral with regard to jurisdictional claims in published maps and institutional affiliations.

Received: 22 May 2018 Accepted: 25 May 2018

Published online: 08 June 2018

\section{References}

1. Moslehi JJ, Salem JE, Sosman JA, et al. Increased reporting of fatal immune checkpoint inhibitor associated myocarditis. Lancet. 2018;391:933.

2. Weisleder $\mathrm{N}, \mathrm{Ma}$ J. Altered $\mathrm{Ca}^{2+}$ sparks in aging skeletal and cardiac muscle. Ageing Res Rev. 2008;7(3):177-88.

3. Cheng H, Lederer WJ. Calcium sparks. Physiol Rev. 2008;88(4):1491-545.

4. Kaneuchi T, Sartain CV, Takeo S, et al. Calcium waves occur as Drosophila oocytes activate. PNAS. 2015;12(3):791-6.

5. Bononi A, Giorgi C, Patergnani S, et al. BAP1 regulates IP3R3-mediated $\mathrm{Ca}^{(2+)}$ flux to mitochondria suppressing cell transformation. Nature. 2017:546(7659):549-53.

6. Kuchay S, Giorgi C, Simoneschi D, et al. PTEN counteracts FBXL2 to promote IP3R3- and $\mathrm{Ca}^{(2+)}$-mediated apoptosis limiting tumour growth. Nature. 2017;546(7659):554-8.

7. Wang L, Xu M, Li Z, et al. Calcium and CaSR/IP3R in prostate cancer development. Cell Biosci. 2018;8:16.

8. Xu M, Seas A, Kiyani M, et al. A temporal examination of calcium signaling in cancer- from tumorigenesis, to immune evasion, and metastasis. Cell Biosci. 2018;8:25.

9. Hulsmans M, Clauss S, Xiao L, et al. Macrophages facilitate electrical conduction in the heart. Cell. 2017;169(3):510-22.

10. Zhou X, Li Z, Wang Z, et al. Syncytium calcium signaling and macrophage function in the heart. Cell Biosci. 2018;8:24.

11. Staehlke $\mathrm{S}$, Rebl H, Finke B, et al. Enhanced calcium ion mobilization in osteoblasts on amino group containing plasma polymer nanolayer. Cell Biosci. 2018:8:22

12. Zhang J, Lakowicz JR. A superior bright NIR luminescent nanoparticle preparation and indicating calcium signaling detection in cells and small animals. Cell Biosci. 2018:8:37. 